\title{
Amorphous alloys - A kind of promising high-performance materials
}

Amorphous alloys, relatively recent metallic materials, exhibit many excellent properties because of their unique structure. They have attracted tremendous attention from both scientists and engineers since their initial discovery 50 years ago. In the early stages, scientists focused on developing new alloy systems with good amorphous structure forming ability and to understanding the fundamentals of the specific atomic packing and structures. It has now been shown that many alloy systems possess the ability to be fabricated into amorphous alloys by rapid solidification technology. In particular, metallic amorphous ribbons can be prepared by melt spinning methods with a cooling rate as high as $1 \times 10^{6} \mathrm{~K} / \mathrm{s}$. This technology made possible wide application of Fe-based magnetic amorphous ribbon in our daily lives. Over the past few decades, bulk amorphous alloys (otherwise known as bulk metallic glasses) have been developed by enhancing the glass forming ability of alloys through adjusting their compositions by trial and error, and also by "magic", or insights that researchers have gained over years of alloy design. Now metallic glasses can be prepared by the copper mold casting method with a relatively low cooling rate. For some alloys the size of metallic glassy samples is as large as 70-80 $\mathrm{mm}$ in diameter and the cooling rate for preparing the glassy sample could be as low as $0.01 \mathrm{~K} / \mathrm{s}$. In addition, many bulk metallic glasses exhibit unusual mechanical and functional properties. For example, Co-based metallic glasses show yield strength of over $6000 \mathrm{MPa}$, the highest strength known in metallic materials. In addition to their impressive performance, metallic glasses possess excellent processing properties, too. Through liquid forming or supercooled liquid forming, both complicated net-shapes and precise nanoscale morphologies of metallic glasses can be fabricated. This suggests that metallic glasses, which possess many excellent mechanical and functional properties, could be potentially used for fabricating nanosized parts or devices, bringing as yet unimagined new contributions to human society.

Metallic glass was invented at California Institute of Technology, but it has become a truly global phenomenon for scientific research and development. The impact of Chinese materials scientists has been enormous: China now is among the most active countries in this field, with the largest number of participating researchers, and Chinese researchers have made important contributions. To honor the memory of the discovery of amorphous alloys half century ago, the Editorial Board of Chinese Science Bulletin invited some of these famous Chinese and overseas Chinese scientists working on amorphous alloys to report and highlight their recent research progress in this special area. The result is a delightful one. We have 17 papers collected in this special issue, which cover broad areas of amorphous alloys, including atomic packing and clusters formation in amorphous alloys [1-3], fragility in glass-forming liquid [4], effects of alloying elements on the glass forming ability of different alloys [5-7], deformation behavior and improved mechanical property of amorphous alloys [8-11], glass-based composites [12,13], high strength metallic glass [14] and functional properties of metallic glasses [15-17].

We would like to sincerely thank the publisher, the journal editors, and all authors for their contributions and effort that have made this special issue possible. We also hope that the reported work in this issue will be intriguing and interesting to scientists all over the world who are working on amorphous alloys.

1 Li Q K, Li M. Rethinking atomic packing and cluster formation in metallic liquids and glasses. Chinese Sci Bull, 2011, 56: 3897-3901

2 Li F W, Qiang J B, Wang Y M, et al. Revisiting Al-Ni-Zr bulk metallic glasses using the 'cluster-resonance' model. Chinese Sci Bull, 2011, 56: 3902-3907

3 Qi L, Liu M, Zhang S L, et al. Atomic packing and short-to-medium range order evolution of Zr-Pd metallic glass. Chinese Sci Bull, 2011, 56: 3908-3911

4 Jia R, Bian X F, Wang Y Y. Thermodynamic determination of fragility in La-based glass-forming liquid. Chinese Sci Bull, 2011, 56: 3912-3918

5 Wang Y, Ding J F, Bai Y W. Influence of B, Al and Nb addition on the glass forming ability and stability of mechanically alloyed $\mathrm{Zr}-\mathrm{Ni}$ amorphous alloys. Chinese Sci Bull, 2011, 56: 3919-3925

6 Li D K, Zhang H F, Wang A M, et al. Effect of Sn addition on the glass-forming ability and mechanical properties of Ni-Nb-Zr bulk metallic glasses. Chinese Sci Bull, 2011, 56: 3926-3931 
7 Wang A D, Zhang M X, Zhang J H, et al. Effect of Ni addition on the glass-forming ability and soft-magnetic properties of FeNiBPNb metallic glasses. Chinese Sci Bull, 2011, 56: 3932-3936

8 Sandor M T, Kecskes L J, He Q, et al. Correlation of mechanical properties in bulk metallic glasses with ${ }^{27}$ Al NMR characteristics. Chinese Sci Bull, 2011, 56: 3937-3941

9 Qiu S B, Gong P, Yao K F. Work toughening effect in $\mathrm{Zr}_{41} \mathrm{Ti}_{14} \mathrm{Cu}_{12.5} \mathrm{Ni}_{10} \mathrm{Be}_{22.5}$ bulk metallic glass. Chinese Sci Bull, 2011, 56: 3942-3947

10 Zhou W, Lu B F, Kong L T, et al. Rolling-induced microstructure change in $\mathrm{Zr}_{65} \mathrm{Al}_{7.5} \mathrm{Ni}_{10} \mathrm{Cu}_{12.5} \mathrm{Ag}_{5}$ bulk metallic glass. Chinese Sci Bull, 2011, 56: 3948-3951

11 Wang G, Mattern N, Bednarčí J, et al. Deformation induced structural evolution in bulk metallic glasses. Chinese Sci Bull, 2011, 56: 3952-3959

$12 \mathrm{Wu} \mathrm{Y}$, Song W L, Zhang Z Y, et al. Relationship between composite structures and compressive properties in CuZr-based bulk metallic glass system. Chinese Sci Bull, 2011, 56: 3960-3964

13 Wei X S, Huang Y J, Vekshin B S, et al. Bulk amorphous $\mathrm{Al}_{85} \mathrm{Ni}_{10} \mathrm{Ce}_{5}$ composite fabricated by cold hydro-mechanical pressing of partially amorphous powders. Chinese Sci Bull, 2011, 56: 3965-3971

14 Zhang T, Yang Q, Ji Y F, et al. Centimeter-scale-diameter Co-based bulk metallic glasses with fracture strength exceeding 5000 MPa. Chinese Sci Bull, 2011, 56: 3972-3977

15 Hui X D, Xu Z Y, Wu Y, et al. Magnetocaloric effect in Er-Al-Co bulk metallic glasses. Chinese Sci Bull, 2011, 56: 3978-3983

16 Wang Y T, Liu Z D, Wang W H. Magnetoresistance oscillations in La-based metallic glass. Chinese Sci Bull, 2011, 56: 3984-3987

17 Zhang C Q, Zhu Z W, Zhang H F, et al. Rapid reductive degradation of azo dyes by a unique structure of amorphous alloys. Chinese Sci Bull, 2011, 56: 3988-3992

YAO KeFu

Special Issue Editor

Department of Mechanical Engineering, Tsinghua University, Beijing 100084, China

Open Access This article is distributed under the terms of the Creative Commons Attribution License which permits any use, distribution, and reproduction in any medium, provided the original author(s) and source are credited. 